\title{
On the role of the syllable in tip-of-the-tongue states
}

\author{
Nina Jeanette Hofferberth \\ Department of Linguistics, Goethe-University Frankfurt, Germany \\ https://doi.org/10.36505/ExLing-2012/05/0015/000221
}

\begin{abstract}
The tip-of-the-tongue (TOT) phenomenon is a type of production failure during phonological encoding. A reaction-time experiment was performed to determine whether the right first syllable of the target facilitates TOT resolution and whether another first syllable inhibits TOT resolution. The syllables were presented individually, which means not in another word related to the target in order to avoid interlopers. It seems that the right first syllable facilitates a positive TOT resolution, while a wrong first syllable has an inhibiting effect. These results indicate that the presentation of the right first syllable of the target word strengthens the weakened phonological connections that cause TOTs and facilitates word retrieval.
\end{abstract}

Key words: speech production, lexical retrieval, word finding failures, first syllable

\section{Theoretical background}

The tip-of-the-tongue (TOT) phenomenon refers to the experience when a familiar word cannot immediately be recalled but "recall is felt to be imminent" (Brown \& McNeill 1966). In the absence of actual retrieval, various aspects of the inaccessible target word are still frequently available, such as first phoneme or letter, first syllable and number of syllables. The recovered partial information can consist of competing items that resemble the target word phonologically and/or semantically and could give rise to competition or conflict during attempts to resolve the TOT. These words that often come to mind while experiencing a TOT and can hamper TOT resolution are so-called "interlopers".

Successful lexical retrieval in a TOT state can be imminent (within minutes) or delayed (after some hours or days) and may occur spontaneously (so-called "pop-ups"). TOT resolution may be achieved through external search strategies (such as looking up the word in a dictionary or by asking someone) or through internal strategies (such as searching in the alphabet or generating similar words). Laboratory studies often involve cueing procedures with a cue-target relationship that is not readily obvious, yet efficient to boost activation of the target word and assist TOT resolution.

Experiments by James \& Burke (2000) demonstrated that phonologically related cues boost activation of the target word. Abrams and colleagues followed up James \& Burke and showed that it is the first syllable of the target (presented in a cue word), which leads to significantly more TOT resolutions, compared to the middle and last syllable (White \& Abrams 2002). Abrams et al. (2003, Experiment 2)

ExLing 2012: Proceedings of 5th Tutorial and Research Workshop on Experimental Linguistics, 27-29 August 2012, Athens, Greece 
demonstrated that TOT resolution requires the entire first syllable and not only the first letter and, furthermore (Experiment 3), that the first syllable (presented in a cue word) significantly improves TOT resolution over the first phoneme - but only when the participants read the cue words silently. Thus, TOT resolution was facilitated by activating the initial syllable via related words sharing that feature.

\begin{abstract}
Aim and scope
In the present study, a reaction-time experiment was performed to determine whether the right first syllable of the target facilitates TOT resolution and whether another first syllable with matched frequency inhibits TOT resolution. The syllables were presented individually, which means not in another word to avoid providing any semantic information of the target and to avoid interlopers. In contrast to the studies of Abrams et al. (2003), the cue did not consist of a related word that shared the same first syllable as the target but only consisted of the first syllable alone.
\end{abstract}

\title{
Experimental Methodology
}

\section{Participants}

The participants in this experiment were ten undergraduates at Heinrich-Heine-University in Dusseldorf who were paid for their services. All students ( 7 female, 3 male) were native speakers of German and between 22 and 30 years old (mean: 25.5 years).

\section{Stimuli}

138 German nouns were presented to induce TOTs. The frequencies of the whole words were taken from DLEX database and the frequencies of the first syllables of these nouns were accessed from the CELEX database (Baayen, Piepenbrock \& Rijn 1993).

\section{Procedure}

Participants read 138 definition-like questions on a computer screen and pushed a button to indicate "know", "don't know" or, "TOT", respectively. When in a TOT state, a written cue was presented. This was either the right first syllable, a wrong syllable with the same CV structure and matched frequency as the fitting syllable, or a neutral baseline condition XXX.

\section{Results}

Of the 138 stimuli, 14 stimuli did not induce TOTs at all. These 14 words were middle- or high-frequent and $50 \%$ had a CVC syllable structure, $42.9 \%$ a CV structure and $7.1 \%$ a VC structure. Of the 124 stimuli that induced TOTs, the most frequent syllable structure was CV (58.1\%), then CVC (16.9\%), VC (15.3\%), CCV (7.3\%) and CCVC (2.4\%). Furthermore, $58.9 \%$ of these were middle-frequent, $33.8 \%$ high-frequent, $6.5 \%$ low-frequent, and $0.8 \%$ without indication from the database. 
The TOT rate was $20.6 \%$ ( $=284$ TOTs, at average 28 TOTs per person). After the cue was presented, $15.5 \%$ of the TOTs could be resolved in the given time of 10 seconds. Of the resolved TOTs, $68.2 \%$ were positive TOTs (answer was consistent with target) and $31.8 \%$ were negative TOTs (answer differed from target).

With the right first syllable, TOTs were positively resolved more often $(19.8 \%)$ in comparison to the control condition $(7.5 \%)$. With a wrong first syllable, TOTs were positively resolved less $(4.3 \%)$ in comparison to the control condition $(7.5 \%)$.

\section{Discussion and conclusion}

Tip-of-the-tongue states are an important source of information concerning the nature of the processes and architecture of the speech production system. TOTs are a unique type of production failure, a breakdown during phonological encoding (cf. Levelt 1989).

TOTs occur about once a week in everyday life and on between 10-20\% of lab stimuli (cf. Brown 2012: 195). In the present study, the TOT rate was $20.6 \%$. With the right first syllable, TOTs were positively resolved more often in comparison to the control condition. With a wrong first syllable, TOTs were positively resolved less in comparison to the control condition. It seems that the right first syllable facilitates a positive TOT resolution, while a wrong first syllable has an inhibiting effect. These results indicate that the presentation of the right first syllable of the target word - presented individually and not in a cue word - strengthens the weakened phonological connections that cause TOTs and facilitates word retrieval.

Only $15.5 \%$ of the TOTs could be resolved in the given time of 10 seconds while the remaining $84.5 \%$ TOTs stayed unresolved. It needs to be discussed if the time span should be longer in order to get more resolved TOTs but then it would be unclear if the participants used other search strategies (such as the semantic one via associations and co-hyponyms) and the syllable cue was not used.

Another reaction-time experiment is currently being run in order to investigate further how the first syllables of the targets, syllable frequencies, and number of cohort words with the same first syllable influence TOT incidence and TOT resolution.

\section{Acknowledgements}

I wish to thank Prof. Dr. Helen Leuniner (Goethe-University Frankfurt) for her support and much inspiring discussion, and Frauke Hellwig (HeinrichHeine-University Dusseldorf) for her help in experiment preparation and programming. 


\section{References}

Abrams, L., White, K.K. and Eitel, S.L. 2003. Isolating phonological components that increase tip-of-the-tongue resolution. Memory \& Cognition 31, 1153-1162.

Brown, A.S. 2012. The tip of the tongue state. New York, Psychology Press.

Brown, R. and McNeill, D. 1966. The 'tip of the tongue' phenomenon. Journal of Verbal Learning and Verbal Behaviour 5, 325-337.

CELEX: Baayen, R.H., Piepenbrock, R. and Rijn, H. 1993. The CELEX lexical database (CD-ROM). Philadelphia, PA, Linguistic Data Consortium, University of Pennsylvania.

DLEX: http://dlexdb.de

James, L. and Burke, D.M. 2000. Phonological priming effects on word retrieval and tip-of-the-tongue experiences in young and older adults. Journal of Experimental Psychology: Learning, Memory, \& Cognition 26, 1378-1391.

Levelt, W.J.M. 1989. Speaking: From intention to articulation. Cambridge, MA, MIT Press.

Meyer, A.S. and Bock, K. 1992. The tip-of-the-tongue phenomenon: Blocking or partial activation? Memory \& Cognition 20, 715-726.

White, K.K. and Abrams, L. 2002. Does priming specific syllables during tip-of-thetongue states facilitate word retrieval in older adults? Psychology and Aging 17, 226-235. 